\title{
On generalized degenerate Gould-Hopper based fully de- generate Bell polynomials
}

\author{
Ugur Duran ${ }^{a, *}$, Mehmet Acikgoz ${ }^{b}$ \\ ${ }^{a}$ Department of the Basic Concepts of Engineering, Faculty of Engineering and Natural Sciences, Iskenderun Technical University, \\ TR-31200 Hatay, Turkey. \\ ${ }^{b}$ Department of Mathematics, Faculty of Science and Arts, University of Gaziantep, TR-27310 Gaziantep, Turkey.
}

\begin{abstract}
In this paper, we introduce both the generalized degenerate Gould-Hopper based degenerate Stirling polynomials of the second kind and the generalized degenerate Gould-Hopper based fully degenerate Bell polynomials. We study and investigate multifarious properties and relations of these polynomials such as explicit formulas, differentiation rules and summation formulas. Moreover, we derive several correlations with the degenerate Bernstein polynomials for these polynomials. Furthermore, we acquire several representations of the generalized degenerate Gould-Hopper based fully degenerate Bell polynomials via not only the fully degenerate Bell polynomials but also the generalized degenerate Gould-Hopper based degenerate Bernoulli, Euler and Genocchi polynomials.
\end{abstract}

Keywords: Degenerate exponential function, Bell polynomials, Gould-Hopper polynomials, Bernstein polynomials, Stirling numbers of the second kind.

2020 MSC: 11B73, 11B68, 11B83, 33B10.

(C)2020 All rights reserved.

\section{Introduction}

The Gould-Hopper polynomials are defined via the following Mac Laurin series expansion (see [1, 11, 14]):

$$
\sum_{n=0}^{\infty} H_{n}^{(j)}(x, y) \frac{t^{n}}{n !}=e^{x t+y t^{j}}
$$

where $j \in \mathbb{N}$ with $j \geqslant 2$. Upon setting $j=1$, the Gould-Hopper polynomials reduce to the representation of the Newton binomial formula. Also, choosing $j=2$ in (1.1), we obtain the familiar Hermite polynomials denoted by $H_{n}(x, y)$ (cf. $\left.[2,10,13,17,28,33,34,37]\right)$.

\footnotetext{
*Corresponding author

Email addresses: mtdrnugur@gmail.com; ugur.duran@iste.edu.tr (Ugur Duran), acikgoz@gantep.edu.tr (Mehmet Acikgoz)
}

doi: $10.22436 /$ jmcs.021.03.07

Received: 2020-01-15 Revised: 2020-02-09 Accepted: 2020-03-03 
The Gould-Hopper polynomials and Hermite polynomials have been used to generalize diverse special polynomials such as Bernoulli, Euler, Bell and Genocchi polynomials (see $[2,10,11,13,14,17,28$, 33, 34]). For instance, Araci et al. [2] introduced a novel concept of the Hermite based Apostol-Genocchi polynomials and investigated several general symmetric identities and implicit summation formulas arising from different analytical means and series manipulation procedure. Duran et al. [13] considered Hermite based poly-Bernoulli polynomials with a q-parameter and gave some properties and relations of them. Duran et al. [14] defined the Gould-Hopper based fully degenerate poly-Bernoulli polynomials with a q-parameter and provided some of their multifarious basic formulas and properties including both addition and difference rule properties. Duran et al. [11] considered generalized degenerate Gould-Hopper polynomials, generalized Gould-Hopper based degenerate central factorial numbers of the second kind and generalized Gould-Hopper based fully degenerate central Bell polynomials via the degenerate exponential functions and then provided several formulae and correlations for these polynomials and numbers related to not only the degenerate Bernstein polynomials but also the Gould-Hopper based fully degenerate Bernoulli, Euler and Genocchi polynomials. Khan [17] defined degenerate Hermite poly-Bernoulli numbers and polynomials and gave some properties and relations. Kurt et al. [28] considered Hermite based Genocchi polynomials. Ozarslan [33] introduced a unified family of Hermite based Apostol-Bernoulli, Euler and Genocchi polynomials and proved a finite series relation between this unification and 3d-Hermite polynomials. Pathan [34] introduced a new kind of generalized Hermite-Bernoulli polynomials and attained some implicit summation formulae and symmetric relations.

For $\lambda \in \mathbb{C}$, the $\lambda$-falling factorial $(x)_{n, \lambda}$ is defined by (see $[3-14,16,17,20-23,25,27,28,34]$ )

$$
(x)_{n, \lambda}= \begin{cases}x(x-\lambda)(x-2 \lambda) \cdots(x-(n-1) \lambda), & n=1,2, \ldots, \\ 1, & n=0 .\end{cases}
$$

In the case $\lambda=1$, the $\lambda$-falling factorial reduces to the familiar falling factorial $(x)_{n}$ (see $[3-14,16,17,20-$ $23,25,27,28,31,33])$

$$
(x)_{n}=x(x-1) \cdots(x-n+1) .
$$

The Stirling numbers of the first kind $S_{1}(n, m)$ are defined by means of the falling factorial as follows

$$
(x)_{n}=\sum_{m=0}^{n} S_{1}(n, m) x^{m}
$$

cf. $[3,12,31,33]$ and see also references cited therein.

The $\Delta_{\lambda}$ difference operator is defined by (see $\left.[10,11,14,31]\right)$

$$
\Delta_{\lambda} f(x)=\frac{1}{\lambda}(f(x+\lambda)-f(x)), \quad \lambda \neq 0 .
$$

The following Lemma will be useful in the derivation of several results.

Lemma $1.1([10,11,14,31])$. The following elementary series manipulation hold:

$$
\sum_{n=0}^{\infty} \sum_{k=0}^{\infty} A(k, n)=\sum_{n=0}^{\infty} \sum_{k=0}^{\lfloor n / j\rfloor} A(k, n-j k),
$$

where $\lfloor\cdot\rfloor$ is the Gauss notation, and represents the maximum integer which does not exceed the number in the square brackets.

The degenerate exponential function $e_{\lambda}^{\chi}(t)$ for a real number $\lambda$ is given by $(c f .[3,5,7,10-14,16,17,20-$ $23,25,27])$

$$
e_{\lambda}^{x}(t)=(1+\lambda t)^{\frac{x}{\lambda}} \text { and } e_{\lambda}^{1}(t)=e_{\lambda}(t) .
$$


It is readily seen that $\lim _{\lambda \rightarrow 0} e_{\lambda}^{x}(t)=e^{x t}$. From (1.2) and (1.4), we obtain the following relation (cf. $[3,5,7,10-14,16,17,20-23,25,27])$

$$
e_{\lambda}^{x}(t)=\sum_{n=0}^{\infty}(x)_{n, \lambda} \frac{t^{n}}{n !},
$$

which satisfies the following difference rule

$$
\Delta_{\lambda} e_{\lambda}^{\chi}(t)=t e_{\lambda}^{x}(t) .
$$

Let $n, j \in \mathbb{Z}$ with $n \geqq 0$ and $j>0$, and let $\lambda_{1}, \lambda_{2} \in \mathbb{R} \backslash\{0\}$. The generalized degenerate Gould-Hopper polynomials $H_{n, \lambda_{1}, \lambda_{2}}^{(j)}(x, y)$ are defined by means of the following generating function (cf. [11]):

$$
\sum_{n=0}^{\infty} H_{n, \lambda_{1}, \lambda_{2}}^{(j)}(x, y) \frac{t^{n}}{n !}=e_{\lambda_{1}}^{x}(t) e_{\lambda_{2}}^{y}\left(t^{j}\right) .
$$

Duran and Acikgoz in [11] investigated diverse formulas and properties of the generalized degenerate Gould-Hopper polynomials $H_{n, \lambda_{2}, \lambda_{3}}^{(j)}(x, y)$.

\section{The generalized degenerate Gould-Hopper based degenerate Stirling polynomials of the second kind}

In this section, we perform to analyze and investigate degenerate forms of some special polynomials and numbers. We focus on the generalized degenerate Gould-Hopper based degenerate Stirling polynomials of the second kind. We then derive several properties and formulas for these polynomials.

For non-negative integer $n$, the Stirling numbers of the second kind $S_{2}(n, m)$ are defined by the following exponential generating function ( $f f .[1-4,7,10-17,20-22,25-27,29-33,38]$

$$
\sum_{n=0}^{\infty} S_{2}(n, m) \frac{t^{n}}{n !}=\frac{\left(e^{t}-1\right)^{m}}{m !}
$$

or by recurrence relation for a fixed non-negative integer $n$ as follows

$$
x^{n}=\sum_{m=0}^{n} S(n, m)(x)_{m} .
$$

For non-negative integer $n$, the degenerate Stirling polynomials of the second kind $S_{2, \lambda}(n, m: x)$ and the degenerate Stirling numbers of the second kind $S_{2, \lambda}(n, m)$ are defined by the following exponential generating functions ( $c f .[19,21,25-27])$

$$
\sum_{n=0}^{\infty} S_{2, \lambda}(n, m: x) \frac{t^{n}}{n !}=\frac{\left(e_{\lambda}(t)-1\right)^{m}}{m !} e_{\lambda}^{x}(t)
$$

and

$$
\sum_{n=0}^{\infty} S_{2, \lambda}(n, m) \frac{t^{n}}{n !}=\frac{\left(e_{\lambda}(t)-1\right)^{m}}{m !} .
$$

When $\lambda$ goes to 0 , the degenerate Stirling numbers of the second kind (2.3) reduce to the Stirling numbers of the second kind (2.1), that is $\lim _{\lambda \rightarrow 0} S_{2, \lambda}(n, m)=S_{2}(n, m)$.

We are now ready to give the definition of the generalized degenerate Gould-Hopper based degenerate Stirling polynomials of the second kind. 
Definition 2.1. Let $\lambda_{1}, \lambda_{2}, \lambda_{3} \in \mathbb{R} \backslash\{0\}$. The generalized degenerate Gould-Hopper based degenerate Stirling polynomials of the second kind $S_{2, \lambda_{1}, \lambda_{2}, \lambda_{3}}^{[j, \omega]}(n, m: x, y)$ are defined by means of the following generating function

$$
\sum_{n=0}^{\infty} S_{2, \lambda_{1}, \lambda_{2}, \lambda_{3}}^{[j, w]}(n, m: x, y) \frac{t^{n}}{n !}=\frac{\left(e_{\lambda_{1}}^{\omega}(t)-1\right)^{m}}{m !} e_{\lambda_{2}}^{x}(t) e_{\lambda_{3}}^{y}\left(t^{j}\right) .
$$

We here analyze some special circumstances of the generalized degenerate Gould-Hopper based degenerate Stirling polynomials of the second kind $S_{2, \lambda_{1}, \lambda_{2}, \lambda_{3}}^{[j, w]}(n, m: x, y)$ as follows.

Remark 2.2.

1. When $x=y=0$, we get the extended degenerate Stirling numbers of the second kind

$$
\sum_{n=0}^{\infty} S_{2, \lambda_{1}, \omega}(n, m) \frac{t^{n}}{n !}=\frac{\left(e_{\lambda_{1}}^{\omega}(t)-1\right)^{m}}{m !} \text {, cf. ([12]). }
$$

2. Upon setting $y=0$, we obtain a new extension of the Stirling polynomials of the second kind, termed unified degenerate Stirling polynomials of the second kind:

$$
\sum_{n=0}^{\infty} S_{2, \lambda_{1}, \lambda_{2}}^{(\omega)}(n, m: x) \frac{t^{n}}{n !}=\frac{\left(e_{\lambda_{1}}^{\omega}(t)-1\right)^{m}}{m !} e_{\lambda_{2}}^{x}(t) .
$$

3. When $x=0$, we attain a novel family of polynomials, which is a generalization of the Stirling polynomials of the second kind:

$$
\sum_{n=0}^{\infty} S_{2, \lambda_{1}, \lambda_{3}}^{[j, w]}(n, m: x) \frac{t^{n}}{n !}=\frac{\left(e_{\lambda_{1}}^{\omega}(t)-1\right)^{m}}{m !} e_{\lambda_{3}}^{x}\left(t^{j}\right) .
$$

4. In the limiting case $\lambda_{1} \rightarrow 0$, generalized Gould-Hopper based degenerate Stirling polynomials of the second kind $S_{2, \lambda_{1}, \lambda_{2}, \lambda_{3}}^{[j, \omega]}(n, m: x, y)$ reduce to the $\omega$-analogue of the degenerate Gould-Hopper based Stirling polynomials of the second kind denoted by $S_{2, w ; \lambda_{2}, \lambda_{3}}^{[j]}(n, m: x, y)$ :

$$
\sum_{n=0}^{\infty} S_{2, \omega ; \lambda_{2}, \lambda_{3}}^{[j]}(n, m: x, y) \frac{t^{n}}{n !}=\frac{\left(e^{\omega t}-1\right)^{m}}{m !} e_{\lambda_{2}}^{x}(t) e_{\lambda_{3}}^{y}\left(t^{j}\right) .
$$

5. When $\omega=1$ and $y=0$ with replacing $\lambda_{2}$ by $\lambda_{1}$, we get the degenerate Stirling polynomials of the second kind $S_{2, \lambda_{1}}(n, m)$ in (2.2), cf. [25-27].

6. Choosing $\omega=1, \lambda_{1}, \lambda_{2} \rightarrow 0$ and $y=0$, we acquire the usual Stirling polynomials of the second kind $\mathrm{S}_{2}(\mathrm{n}, \mathrm{m}: \mathrm{x})$, cf. $[12,19,24-27]$.

7. When $\lambda_{1} \rightarrow 0, w=1$ and $x=y=0$, we attain the familiar Stirling numbers of the second kind $\mathrm{S}_{2}(\mathrm{n}, \mathrm{m})$ in $(2.1)$, cf. $[1-4,7,10-17,20-22,25-27,29-33,38]$.

Proposition 2.3. The following summation formulas

$$
\begin{aligned}
& S_{2, \lambda_{1}, \lambda_{2}, \lambda_{3}}^{[j, w]}(n, m: x, y)=\sum_{s=0}^{n}\left(\begin{array}{l}
n \\
s
\end{array}\right) S_{2, \lambda_{1}, \omega}(s, m) H_{n-s, \lambda_{2}, \lambda_{3}}^{(j)}(x, y), \\
& S_{2, \lambda_{1}, \lambda_{2}, \lambda_{3}}^{[j, \omega]}(n, m: x, y)=\sum_{s=0}^{n}\left(\begin{array}{l}
n \\
s
\end{array}\right)(x)_{s, \lambda_{2}} S_{2, \lambda_{1}, \lambda_{3}}^{[j, \omega]}(n-s, m: y),
\end{aligned}
$$




$$
S_{2, \lambda_{1}, \lambda_{2}, \lambda_{3}}^{[j, w]}(n, m: x, y)=n ! \sum_{s=0}^{\left\lfloor\frac{n}{j}\right\rfloor} \frac{(y)_{s, \lambda_{3}} S_{2, \lambda_{1}, \lambda_{2}, \lambda_{3}}^{(w)}(n-s j, m: x)}{(n-j s) ! s !}
$$

hold true.

Proposition 2.4. For $k, m, n \in \mathbb{N}$ and $\lambda_{1}, \lambda_{2}, \lambda_{3} \in \mathbb{R} \backslash\{0\}$, we have

$$
\begin{aligned}
& S_{2, \lambda_{1}, \lambda_{2}, \lambda_{3}}^{[j, w]}(n, m+k: x, y)=\frac{k ! m !}{(k+m) !} \sum_{u=0}^{n}\left(\begin{array}{l}
n \\
u
\end{array}\right) S_{2, \lambda_{1}, \lambda_{2}, \lambda_{3}}^{[j, w]}(u, m: x, y) S_{2, \lambda_{1}, w}(n-u, k), \\
& S_{2, \lambda_{1}, \lambda_{2}, \lambda_{3}}^{[j, w]}(n+m: k, y)=\frac{k ! m !}{(k+m) !} \sum_{u=0}^{n}\left(\begin{array}{l}
n \\
u
\end{array}\right) S_{2, \lambda_{1}, \lambda_{2}, \lambda_{3}}^{(\omega)}(u, m: x) S_{2, \lambda_{1}, \lambda_{3}}^{[j, w]}(n-u, k: y) .
\end{aligned}
$$

Here is a differentiation rule for the generalized degenerate Gould-Hopper based degenerate Stirling polynomials of the second kind $S_{2, \lambda_{1}, \lambda_{2}, \lambda_{3}}^{[j, \omega]}(n, m: x, y)$.

Theorem 2.5. The following relation

$$
\begin{aligned}
\frac{\partial}{\partial \omega} S_{2, \lambda_{1}, \lambda_{2}, \lambda_{3}}^{[j, \omega]}(n, m: x, y)= & \frac{n !}{(n-u) !} \sum_{s=0}^{n} \sum_{k=0}^{m} \sum_{u=1}^{\infty}\left(\begin{array}{c}
n \\
s
\end{array}\right)\left(\begin{array}{c}
m \\
k
\end{array}\right) \frac{(-1)^{\mathfrak{u}+k+1}}{m !} \lambda_{1}^{u-1} \\
& \times \frac{m-k}{u}(\omega(m-k))_{n-\mathfrak{u}-s, \lambda_{1}} H_{s, \lambda_{2}, \lambda_{3}}^{(j)}(x, y)
\end{aligned}
$$

holds true for $\lambda_{1}, \lambda_{2}, \lambda_{3} \in \mathbb{R} \backslash\{0\}$.

Proof. In view of (2.4), we get

$$
\begin{aligned}
\frac{\partial}{\partial \omega} & \sum_{n=0}^{\infty} S_{2, \lambda_{1}, \lambda_{2}, \lambda_{3}}^{[j, \omega]}(n: x, y) \frac{t^{n}}{n !} \\
& =\frac{\partial}{\partial \omega} \frac{\left(e_{\lambda_{1}}^{\omega}(t)-1\right)^{m}}{m !} e_{\lambda_{2}}^{x}(t) e_{\lambda_{3}}^{y}\left(t^{j}\right) \\
& =\sum_{k=0}^{m}\left(\begin{array}{c}
m \\
k
\end{array}\right)(-1)^{k} \frac{e_{\lambda_{2}}^{x}(t) e_{\lambda_{3}}^{y}\left(t^{j}\right)}{m !}\left(1+\lambda_{1} t\right)^{\frac{\omega(m-k)}{\lambda_{1}}} \ln \left(1+\lambda_{1} t\right)^{\frac{m-k}{\lambda_{1}}} \\
& =\sum_{k=0}^{m}\left(\begin{array}{c}
m \\
k
\end{array}\right) \sum_{u=1}^{\infty} \frac{(-1)^{u+k+1}}{m !} \frac{m-k}{u} \lambda_{1}^{\mathfrak{u}-1} t^{u}\left(1+\lambda_{1} t\right)^{\frac{\omega(m-k)}{\lambda_{1}}} e_{\lambda_{2}}^{x}(t) e_{\lambda_{3}}^{y}\left(t^{j}\right) \\
& =\sum_{n=0}^{\infty} \sum_{s=0}^{n} \sum_{k=0}^{m} \sum_{u=1}^{\infty}\left(\begin{array}{c}
n \\
s
\end{array}\right)\left(\begin{array}{c}
m \\
k
\end{array}\right) \frac{(-1)^{u+k+1}}{m !} \frac{m-k}{u} \lambda_{1}^{u-1}(\omega(m-k))_{n-s, \lambda_{1}} H_{s, \lambda_{2}, \lambda_{3}}^{(j)}(x, y) \frac{t^{n+u}}{n !},
\end{aligned}
$$

which implies the claimed result (2.6).

Theorem 2.6. The following identity

$$
\frac{\partial}{\partial x} S_{2, \lambda_{1}, \lambda_{2}, \lambda_{3}}^{[j, w]}(n, m: x, y)=\frac{n !}{(n-u) !} \sum_{u=1}^{\infty} \frac{(-1)^{u+1}}{u} \lambda_{2} S_{2, \lambda_{1}, \lambda_{2}, \lambda_{3}}^{[j, w]}(n-u, m: x, y)
$$

is valid for $\lambda_{1}, \lambda_{2}, \lambda_{3} \in \mathbb{R} \backslash\{0\}$.

Proof. From (2.4), the asserted result (2.7) can be obtained by utilizing similar method used in the proof of Theorem 2.5. Thus, we omit the proof. 
Theorem 2.7. For $\lambda_{1}, \lambda_{2}, \lambda_{3} \in \mathbb{R} \backslash\{0\}$, we have

$$
\frac{\partial}{\partial y} S_{2, \lambda_{1}, \lambda_{2}, \lambda_{3}}^{[j, w]}(n, m: x, y)=\frac{n !}{(n-j u) !} \sum_{u=1}^{\infty} \frac{(-1)^{u+1} \lambda_{3}^{u-1}}{u} S_{2, \lambda_{1}, \lambda_{2}, \lambda_{3}}^{[j, w]}(n-j u, m: x, y) .
$$

Proof. From (2.4), the asserted result (2.8) can be similarly obtained by utilizing same method used in the proof of Theorem 2.5. Thus, we omit the proof.

We here give the following correlation.

Theorem 2.8. The following relation

$$
S_{2, \lambda_{1}, \lambda_{2}, \lambda_{3}}^{[j, \omega]}(n, m: x, y)=\sum_{k=0}^{n} \sum_{u=0}^{\infty}\left(\begin{array}{l}
n \\
k
\end{array}\right) \frac{x !}{(x-u) !} S_{2, \lambda_{2}}(n-k, u) S_{2, \lambda_{1}, \lambda_{3}}^{[j, \omega]}(k, m: y)
$$

holds true for $\lambda_{1}, \lambda_{2}, \lambda_{3} \in \mathbb{R} \backslash\{0\}$.

Proof. By Definition 2.1, we have

$$
\begin{aligned}
\sum_{n=0}^{\infty} S_{2, \lambda_{1}, \lambda_{2}, \lambda_{3}}^{[j, w]}(n, m: x, y) \frac{t^{n}}{n !} & =\left(e_{\lambda_{2}}(t)-1+1\right)^{x} \frac{\left(e_{\lambda_{1}}^{\omega}(t)-1\right)^{m}}{m !} e_{\lambda_{3}}^{y}\left(t^{j}\right) \\
& =\sum_{u=0}^{\infty}\left(\begin{array}{l}
x \\
u
\end{array}\right)\left(e_{\lambda_{2}}(t)-1\right)^{u} \frac{\left(e_{\lambda_{1}}^{\omega}(t)-1\right)^{m}}{m !} e_{\lambda_{3}}^{y}\left(t^{j}\right) \\
& =\sum_{n=0}^{\infty} \sum_{k=0}^{n} \sum_{u=0}^{\infty}\left(\begin{array}{l}
n \\
k
\end{array}\right) \frac{x !}{(x-u) !} S_{2, \lambda_{2}}(n-k, u) S_{2, \lambda_{1}, \lambda_{3}}^{[j, w]}(k, m: y) \frac{t^{n}}{n !},
\end{aligned}
$$

which implies the desired result (2.9).

We here give the following correlation.

Theorem 2.9. The following correlation

$$
S_{2, \lambda_{1}, \lambda_{2}, \lambda_{3}}^{[j, w]}(n, m: x, y)=\sum_{l=0}^{n} S_{2, w ; \lambda_{2}, \lambda_{3}}^{[j]}(l, m: x, y) S_{1}(n, l) \lambda_{1}^{n-l}
$$

is valid for $\lambda_{1}, \lambda_{2}, \lambda_{3} \in \mathbb{R} \backslash\{0\}$.

Proof. By Definition 2.1 and the identity (1.4), we obtain

$$
\begin{aligned}
\sum_{n=0}^{\infty} S_{2, \lambda_{1}, \lambda_{2}, \lambda_{3}}^{[j, w]}(n, m: x, y) \frac{t^{n}}{n !} & =\sum_{1=0}^{\infty} S_{2, \omega ; \lambda_{2}, \lambda_{3}}^{[j]}(l, m: x, y) \lambda_{1}^{-l} \frac{\left(\log \left(1+\lambda_{1} t\right)\right)^{l}}{l !} \\
& =\sum_{n=0}^{\infty} \sum_{l=0}^{n} S_{2, \omega ; \lambda_{2}, \lambda_{3}}^{[j]}(l, m: x, y) S_{1}(n, l) \lambda_{1}^{n-l} \frac{t^{n}}{n !}
\end{aligned}
$$

which provides the desired result (2.10).

\section{The generalized degenerate Gould-Hopper based fully degenerate Bell polynomials}

In this section, we consider a new concept of the degenerate Bell polynomials by using the generalized degenerate Gould-Hopper polynomials. We then get several formulas and identities for these polynomials such as summation formula, explicit formula and differentiation property. 
The classical Bell polynomials $B_{n}(x)$ (also called exponential polynomials) are defined by means of the following generating function: (cf. $[1,2,12,14-16,19-22,26,31,32])$

$$
\sum_{n=0}^{\infty} B_{n}(x) \frac{t^{n}}{n !}=e^{x\left(e^{t}-1\right)}
$$

The classical Bell numbers $B_{n}$ are determined by taking $x=1$ in (3.1), that is $B_{n}(1):=B_{n}$ and are given by the following exponential generating function:

$$
\sum_{n=0}^{\infty} B_{n} \frac{t^{n}}{n !}=e^{\left(e^{t}-1\right)}
$$

The Bell polynomials introduced by Bell [3] appear as a standard mathematical tool and arise in combinatorial analysis. The familiar Bell polynomials have been intensely investigated by several mathematicians, cf. $[1,2,12,14-16,19-22,26,31,32]$ and see also the references cited therein.

The usual Bell polynomials and Stirling numbers of the second kind satisfy the following relation ( $c f$. $[1,2,14,15,20,26,32])$

$$
B_{n}(x)=\sum_{m=0}^{n} S_{2}(n, m) x^{m}
$$

The degenerate Bell polynomials are given by the following Taylor series expansion at $t=0$ as follows (cf. $[11,21,23-25,27])$

$$
\sum_{n=0}^{\infty} B_{n, \lambda}(x) \frac{t^{n}}{n !}=e^{x\left(e_{\lambda}(t)-1\right)}
$$

When $x=1$ in (3.4), the polynomials $B_{n, \lambda}(x)$ reduce to the degenerate Bell numbers $B_{n, \lambda}(1):=B_{n, \lambda}$ having the following generating function

$$
\sum_{n=0}^{\infty} B_{n, \lambda} \frac{t^{n}}{n !}=e^{\left(e_{\lambda}(t)-1\right)}
$$

We note that using (1.4), the degenerate classical Bell polynomials (3.4) reduce the classical Bell polynomials in the following limit cases:

$$
\lim _{\lambda \rightarrow 0} B_{n, \lambda}(x)=B_{n}(x) .
$$

The degenerate Bell polynomials and the degenerate Stirling numbers of the second kind satisfy the following relation ( $c f .[23-25,27])$

$$
\mathrm{B}_{\mathrm{n}, \lambda}(x)=\sum_{m=0}^{\mathrm{n}} \mathrm{S}_{2, \lambda}(\mathrm{n}, \mathrm{m}) \mathrm{x}^{\mathrm{m}}
$$

We are now ready to define the generalized degenerate Gould-Hopper based fully degenerate Bell polynomials and numbers by the following Definition 3.1.

Definition 3.1. Let $\lambda, \lambda_{1}, \lambda_{2}, \lambda_{3} \in \mathbb{R} \backslash\{0\}$. The generalized degenerate Gould-Hopper based fully degenerate Bell polynomials $B_{n, \lambda, \lambda, \lambda_{2}, \lambda_{3}}^{(c, j)}(z: \omega, x, y)$ are defined by the following exponential generating function

$$
\sum_{n=0}^{\infty} B_{n, \lambda, \lambda_{1}, \lambda_{2}, \lambda_{3}}^{[j]}(z: \omega, x, y) \frac{t^{n}}{n !}=e_{\lambda}^{z}\left(e_{\lambda_{1}}^{\omega}(t)-1\right) e_{\lambda_{2}}^{x}(t) e_{\lambda_{3}}^{y}\left(t^{j}\right) .
$$


Upon setting $z=1$, the generalized degenerate Gould-Hopper based fully degenerate Bell polynomials reduce to the corresponding numbers $B_{n, \lambda, \lambda_{1}, \lambda_{2}, \lambda_{3}}^{[j]}(1: \omega, x, y):=B_{n, \lambda, \lambda_{1}, \lambda_{2}, \lambda_{3}}^{[j]}(\omega, x, y)$ termed as generalized degenerate Gould-Hopper based fully degenerate Bell numbers:

$$
\sum_{n=0}^{\infty} B_{n, \lambda, \lambda_{1}, \lambda_{2}, \lambda_{3}}^{[j]}(\omega, x, y) \frac{t^{n}}{n !}=e_{\lambda}\left(e_{\lambda_{1}}^{\omega}(t)-1\right) e_{\lambda_{2}}^{x}(t) e_{\lambda_{3}}^{y}\left(t^{j}\right) .
$$

We now examine diverse special cases of the generalized degenerate Gould-Hopper based fully degenerate Bell polynomials as follows.

\section{Remark 3.2.}

1. When $\omega=1$, the polynomials $B_{n, \lambda, \lambda_{1}, \lambda_{2}, \lambda_{3}}^{[j]}(z: \omega, x, y)$ in (3.7) reduce to the generalized GouldHopper based degenerate Bell polynomials $B_{n, \lambda, \lambda_{1}, \lambda_{2}, \lambda_{3}}^{[j]}(z: x, y)$ are as in (3.9), which are also new generalizations of the Bell polynomials $B_{n}(x)$ in (3.1), given by

$$
\sum_{n=0}^{\infty} B_{n, \lambda, \lambda_{1}, \lambda_{2}, \lambda_{3}}^{[j]}(z: x, y) \frac{t^{n}}{n !}=e_{\lambda}^{z}\left(e_{\lambda_{1}}(t)-1\right) e_{\lambda_{2}}^{x}(t) e_{\lambda_{3}}^{y}\left(t^{j}\right) .
$$

2. Upon setting $\lambda, \lambda_{2}, \lambda_{3} \rightarrow 0$, we get the Gould-Hopper based generalized degenerate Bell polynomials $B_{n, \lambda_{1}}^{[j]}(z: \omega, x, y)(3.10)$, which are extensions of the Bell polynomials (3.1), shown by

$$
\sum_{n=0}^{\infty} B_{n, \lambda_{1}}^{[j]}(z: \omega, x, y) \frac{t^{n}}{n !}=e^{z\left(e_{\lambda_{1}}^{\omega}(t)-1\right)} e^{x t+y t^{j}}
$$

3. Choosing $y=x=0$, we obtain a new generalization of the degenerate Bell polynomials given below:

$$
\sum_{n=0}^{\infty} B_{n, \lambda, \lambda_{1}}(z: \omega) \frac{t^{n}}{n !}=e_{\lambda}^{z}\left(e_{\lambda_{1}}^{\omega}(t)-1\right) .
$$

4. Setting $\omega=1, y=x=0$ and $\lambda_{1} \rightarrow 0$, we attain the degenerate Bell polynomials and numbers denoted by $\mathcal{B}_{n, \lambda}(z)$ and $\mathcal{B}_{n, \lambda}$, which is different from the polynomials and numbers in (3.4) and (3.5) given by Kim et al. [21]:

$$
\sum_{n=0}^{\infty} \mathcal{B}_{n, \lambda}(z) \frac{t^{n}}{n !}=e_{\lambda}^{z}\left(e^{t}-1\right) \text { and } \sum_{n=0}^{\infty} \mathcal{B}_{n, \lambda} \frac{t^{n}}{n !}=e_{\lambda}\left(e^{t}-1\right) .
$$

5. In the special case $\lambda, \lambda_{1}, \lambda_{2}, \lambda_{3} \rightarrow 0$, we acquire Gould-Hopper based extended Bell polynomials $\mathrm{B}_{\mathrm{n}, \mathrm{w}}^{(\mathrm{j})}(x: x, y)$ as follows

$$
\sum_{n=0}^{\infty} B_{n, \omega}^{(j)}(z: x, y) \frac{t^{n}}{n !}=e^{z\left(e^{\omega t}-1\right)} e^{x t+y t^{j}}
$$

6. When $\omega=1, y=x=0$ and $\lambda \rightarrow 0$, we obtain the degenerate Bell polynomials and numbers in (3.4) and (3.5) (cf. [11, 21, 23-25, 27]).

7. When $\lambda, \lambda_{1} \rightarrow 0, y=x=0$ and $\omega=1$, we arrive at the usual Bell polynomials and numbers in (3.1) and (3.2) (cf. $[1,2,14,15,20,26,32])$.

We now investigate some properties and formulas of the generalized degenerate Gould-Hopper based fully degenerate Bell polynomials $B_{n, \lambda, \lambda_{1}, \lambda_{2}, \lambda_{3}}^{(c, j)}(z: \omega, x, y)$. Hence, we firstly provide the following theorem. 
Theorem 3.3. For $\lambda, \lambda_{1}, \lambda_{2}, \lambda_{3} \in \mathbb{R} \backslash\{0\}$, we have

$$
B_{n, \lambda, \lambda_{1}, \lambda_{2}, \lambda_{3}}^{[j]}(z: \omega, x, y)=\sum_{l=0}^{n}\left(\begin{array}{l}
n \\
l
\end{array}\right) B_{l, \lambda, \lambda_{1}}(z: \omega) H_{n-l, \lambda_{2}, \lambda_{3}}^{(j)}(x, y) .
$$

Proof. By (1.6) and (3.7), the asserted result (3.11) can be directly derived by using Cauchy product. Hence, we omit the proof.

A generalization of the well-known relations in (3.3) and (3.6) is given below.

Theorem 3.4. The following relation

$$
B_{n, \lambda, \lambda_{1}, \lambda_{2}, \lambda_{3}}^{[j]}(z: \omega, x, y)=\sum_{m=0}^{n} S_{2, \lambda_{1}, \lambda_{2}, \lambda_{3}}^{[j, \omega]}(n: m: x, y)(z)_{m, \lambda}
$$

holds true for $\lambda, \lambda_{1}, \lambda_{2}, \lambda_{3} \in \mathbb{R} \backslash\{0\}$.

Proof. By Definition 3.1 and formulas (1.4) and (1.5), the claimed result (3.12) can be directly obtained by utilizing Cauchy product. Thus, we omit the proof.

We now state a summation formula for $B_{n, \lambda, \lambda_{1}, \lambda_{2}, \lambda_{3}}^{[j]}(z: \omega, x, y)$ as follows.

Theorem 3.5. The following summation formula

$$
B_{n, \lambda, \lambda_{1}, \lambda_{2}, \lambda_{3}}^{[j]}\left(z_{1}+z_{2}: \omega, x, y\right)=\sum_{m=0}^{n}\left(\begin{array}{l}
n \\
m
\end{array}\right) B_{n-m, \lambda, \lambda_{1}, \lambda_{2}, \lambda_{3}}^{[j]}\left(z_{1}: \omega, x, y\right) B_{m, \lambda, \lambda_{1}}\left(z_{2}: \omega\right),
$$

is valid for $\lambda, \lambda_{1}, \lambda_{2}, \lambda_{3} \in \mathbb{R} \backslash\{0\}$.

Proof. By Definition 3.1 and the identity (1.5), the desired result (3.13) can be directly acquired by using Cauchy product. Hence, we omit the proof.

We now provide a correlation as follows.

Theorem 3.6. The following formula

$$
B_{n, \lambda, \lambda_{1}, \lambda_{2}, \lambda_{3}}^{[j]}(z: \omega, x, y)=\sum_{m=0}^{n} \sum_{l=0}^{n}(z)_{m, \lambda} S_{2, \omega ; \lambda_{2}, \lambda_{3}}^{[j]}(l, m: x, y) S_{1}(n, l) \lambda_{1}^{n-l}
$$

holds true for $\lambda, \lambda_{1}, \lambda_{2}, \lambda_{3} \in \mathbb{R} \backslash\{0\}$.

Proof. By Definition 3.1 and Theorem 2.9, we get

$$
\begin{aligned}
\sum_{n=0}^{\infty} B_{n, \lambda, \lambda_{1}, \lambda_{2}, \lambda_{3}}^{[j]}(z: \omega, x, y) \frac{t^{n}}{n !} & =\sum_{m=0}^{\infty}(z)_{m, \lambda} \frac{\left(e_{\lambda_{1}}^{\omega}(t)-1\right)^{m}}{m !} e_{\lambda_{2}}^{x}(t) e_{\lambda_{3}}^{y}\left(t^{j}\right) \\
& =\sum_{m=0}^{\infty}(z)_{m, \lambda} \sum_{n=0}^{\infty} \sum_{l=0}^{n} S_{2, \omega ; \lambda_{2}, \lambda_{3}}^{[j]}(l, m: x, y) S_{1}(n, l) \lambda_{1}^{n-l} \frac{t^{n}}{n !} \\
& =\sum_{n=0}^{\infty} \sum_{m=0}^{n} \sum_{l=0}^{n}(z)_{m, \lambda} S_{2, \omega ; \lambda_{2}, \lambda_{3}}^{[j]}(l, m: x, y) S_{1}(n, l) \lambda_{1}^{n-l} \frac{t^{n}}{n !}
\end{aligned}
$$

which gives the claimed result (3.14). 
We here provide an explicit formula for $B_{n, \lambda, \lambda_{1}, \lambda_{2}, \lambda_{3}}^{[j]}(z: \omega, x, y)$ as follows.

Theorem 3.7. The following explicit formula

$$
\begin{aligned}
B_{n, \lambda, \lambda_{1}, \lambda_{2}, \lambda_{3}}^{[j]}(z: \omega, x, y)= & \sum_{u=0}^{n} \sum_{m=0}^{\infty} \sum_{k=0}^{m} \sum_{k=0}^{\lfloor(n-u) / j\rfloor}\left(\begin{array}{l}
n \\
u
\end{array}\right)\left(\begin{array}{l}
m \\
k
\end{array}\right)(-1)^{m-k} \frac{(n-u) !}{m !} \\
& \times(z)_{m, \lambda}(\omega k)_{u, \lambda_{1}} \frac{(x)_{n-\mathfrak{u}-j k, \lambda_{2}}(y)_{k, \lambda_{3}}}{(n-\mathfrak{u}-j k) ! k !}
\end{aligned}
$$

holds true for $\lambda, \lambda_{1}, \lambda_{2}, \lambda_{3} \in \mathbb{R} \backslash\{0\}$.

Proof. By Definition 3.1 and formulas (1.3) and (1.5), we get

$$
\begin{aligned}
& \sum_{n=0}^{\infty} B_{n, \lambda, \lambda_{1}, \lambda_{2}, \lambda_{3}}^{[j]}(z: \omega, x, y) \frac{t^{n}}{n !} \\
& \quad=\sum_{m=0}^{\infty}(z)_{m, \lambda} \frac{\left(e_{\lambda_{1}}^{\omega}(t)-1\right)^{m}}{m !} e_{\lambda_{2}}^{x}(t) e_{\lambda_{3}}^{y}\left(t^{j}\right) \\
& \quad=\sum_{m=0}^{\infty} \frac{(z)_{m, \lambda}}{m !} \sum_{k=0}^{m}\left(\begin{array}{c}
m \\
k
\end{array}\right)(-1)^{m-k}\left(1+\lambda_{1} t\right)^{\frac{\omega k}{\lambda_{1}}}\left(1+\lambda_{2} t\right)^{\frac{x}{\lambda_{2}}}\left(1+\lambda_{3} t^{j}\right)^{\frac{y}{\lambda_{3}}} \\
& \quad=\sum_{n=0}^{\infty}\left(\sum_{m=0}^{\infty} \sum_{k=0}^{m}\left(\begin{array}{c}
m \\
k
\end{array}\right)(z)_{m, \lambda} \frac{(-1)^{m-k}}{m !}(\omega k)_{n, \lambda_{1}}\right) \frac{t^{n}}{n !} \sum_{n=0}^{\infty}\left(n ! \sum_{k=0}^{\lfloor n / j\rfloor} \frac{(x)_{n-j k, \lambda_{2}}(y)_{k, \lambda}}{(n-j k) ! k !}\right) \frac{t^{n}}{n !},
\end{aligned}
$$

which gives the asserted result (3.15).

We now present the following derivation property with respect to $z$ for $B_{n, \lambda, \lambda_{1}, \lambda_{2}, \lambda_{3}}^{[j]}(z: \omega, x, y)$.

Theorem 3.8. The following relation

$$
\frac{d}{d z} B_{n, \lambda, \lambda_{1}, \lambda_{2}, \lambda_{3}}^{[j]}(z: \omega, x, y)=\sum_{u=0}^{n} \sum_{m=1}^{\infty}\left(\begin{array}{l}
n \\
u
\end{array}\right) B_{n-u, \lambda, \lambda_{1}, \lambda_{2}, \lambda_{3}}^{[j]}(z: \omega, x, y) S_{2, \lambda_{1} ; w}(u, m)(m-1) !(-\lambda)^{m-1}
$$

holds true for $\lambda, \lambda_{1}, \lambda_{2}, \lambda_{3} \in \mathbb{R} \backslash\{0\}$.

Proof. By Definition 3.1 and formulas (1.4) and (1.5), we get

$$
\begin{aligned}
\frac{d}{d z} & \sum_{n=0}^{\infty} B_{n, \lambda, \lambda_{1}, \lambda_{2}, \lambda_{3}}^{[j]}(z: \omega, x, y) \frac{t^{n}}{n !} \\
& =\frac{d}{d x} e_{\lambda}^{z}\left(e_{\lambda_{1}}^{\omega}(t)-1\right) e_{\lambda_{2}}^{x}(t) e_{\lambda_{3}}^{y}\left(t^{j}\right) \\
& =\left(1+\lambda\left(e_{\lambda_{1}}^{\omega}(t)-1\right)\right)^{\frac{z}{\lambda}} \ln \left(\left(1+\lambda\left(e_{\lambda_{1}}^{\omega}(t)-1\right)\right)^{\lambda^{-1}}\right) e_{\lambda_{2}}^{x}(t) e_{\lambda_{3}}^{y}\left(t^{j}\right) \\
& =\sum_{n=0}^{\infty} B_{n, \lambda, \lambda_{1}, \lambda_{2}, \lambda_{3}}^{[j]}(z: \omega, x, y) \frac{t^{n}}{n !} \sum_{m=1}^{\infty}(m-1) !(-\lambda)^{m-1} \frac{\left(e_{\lambda_{1}}^{\omega}(t)-1\right)^{m}}{m !} \\
& =\sum_{n=0}^{\infty} \sum_{u=0}^{n}\left(\begin{array}{l}
n \\
u
\end{array}\right) \sum_{m=1}^{\infty}(m-1) !(-\lambda)^{m-1} B_{n-u, \lambda, \lambda_{1}, \lambda_{2}, \lambda_{3}}^{[j]}(z: w, x, y) S_{2, \lambda_{1} ; \omega}(u, m) \frac{t^{n}}{n !},
\end{aligned}
$$

which means the claimed result (3.16). 


\section{Multifarious connected formulas}

In this section, we perform to get several diverse relations for $B_{n, \lambda, \lambda_{1}, \lambda_{2}, \lambda_{3}}^{[j]}(x: \omega, x, y)$ with some other degenerate polynomials including both the degenerate Bernstein polynomials and the generalized degenerate Gould-Hopper based degenerate Bernoulli, Genocchi, and Euler polynomials.

Kim and Kim [22] defined the degenerate Bernstein polynomials by means of the following generating function:

$$
\sum_{n=0}^{\infty} \mathfrak{B}_{k, n}(x: \lambda) \frac{t^{n}}{n !}=\frac{(x)_{k, \lambda}}{k !} t^{k} e_{\lambda}^{1-x}(t) .
$$

By utilizing (1.6), (3.7), and (4.1), we consider that

$$
\begin{aligned}
\sum_{n=0}^{\infty} B_{n, \lambda, \lambda_{1}, \lambda_{2}, \lambda_{3}}^{[j]}(z: \omega, x, y) \frac{t^{n}}{n !} & \\
& =\sum_{m=0}^{\infty}(z)_{m, \lambda} \frac{\left(e_{\lambda_{1}}^{\omega}(t)-1\right)^{m}}{m !} e_{\lambda_{2}}^{x}(t) e_{\lambda_{3}}^{y}\left(t^{j}\right) \\
& =\sum_{m=0}^{\infty} \sum_{k=0}^{m} \frac{(z)_{m, \lambda}}{t^{k}(1-\omega k)_{k, \lambda_{1}}} \frac{(-1)^{m-k}}{(m-k) !} \frac{(1-\omega k)_{k, \lambda_{1}}}{k !} t^{k}\left(1+\lambda_{1} t\right)^{\frac{\omega k}{\lambda_{1}}} e_{\lambda_{2}}^{x}(t) e_{\lambda_{3}}^{y}\left(t^{j}\right) \\
& =\sum_{m=0}^{\infty} \sum_{k=0}^{m} \frac{(z)_{m, \lambda}}{t^{k}(1-\omega k)_{k, \lambda_{1}}} \frac{(-1)^{m-k}}{(m-k) !} \sum_{n=0}^{\infty} \sum_{u=0}^{n}\left(\begin{array}{l}
n \\
u
\end{array}\right) \mathfrak{B}_{k, u}\left(1-\omega k: \lambda_{1}\right) H_{n-u, \lambda_{2}, \lambda_{3}}^{(j)}(x, y) \frac{t^{n-k}}{n !}
\end{aligned}
$$

Hence, we arrive at the following theorem.

Theorem 4.1. The following correlation

$$
\begin{aligned}
\mathrm{B}_{n, \lambda, \lambda_{1}, \lambda_{2}, \lambda_{3}}^{[j]}(z: \omega, x, y)= & n ! \sum_{m=0}^{\infty} \sum_{k=0}^{m} \frac{(z)_{m, \lambda}}{(1-\omega k)_{k, \lambda_{1}}} \frac{(-1)^{m-k}}{(m-k) !(n+k) !} \\
& \times \sum_{u=0}^{n+k}\left(\begin{array}{c}
n+k \\
u
\end{array}\right) \mathfrak{B}_{k, u}\left(1-\omega k: \lambda_{1}\right) H_{n+k-u, \lambda_{2}, \lambda_{3}}^{(j)}(x, y)
\end{aligned}
$$

holds true.

Let

$$
\Upsilon=\frac{(z)_{k, \lambda}}{k !}\left(e_{\lambda_{1}}^{\omega}(t)-1\right)^{k} e_{\lambda}^{1-z}\left(e_{\lambda_{1}}^{\omega}(t)-1\right) e_{\lambda_{2}}^{x}(t) e_{\lambda_{3}}^{y}\left(t^{j}\right)
$$

From (2.4) and (4.1), we obtain

$$
\begin{aligned}
\Upsilon & =\sum_{\mathfrak{u}=0}^{\infty} \mathfrak{B}_{k, u}(z: \lambda) \frac{\left(e_{\lambda_{1}}^{\omega}(t)-1\right)^{u}}{u !} e_{\lambda_{2}}^{x}(t) e_{\lambda_{3}}^{y}\left(t^{j}\right) \\
& =\sum_{\mathfrak{u}=0}^{\infty} \mathfrak{B}_{k, u}(z: \lambda) \sum_{n=0}^{\infty} S_{2, \lambda_{1}, \lambda_{2}, \lambda_{3}}^{[j, w]}(n, u: x, y) \frac{t^{n}}{n !} \\
& =\sum_{n=0}^{\infty}\left(\sum_{\mathfrak{u}=0}^{n} \mathfrak{B}_{k, u}(z: \lambda) S_{2, \lambda_{1}, \lambda_{2}, \lambda_{3}}^{[j, w]}(n, u: x, y)\right) \frac{t^{n}}{n !}
\end{aligned}
$$

and on the other hand, by (2.5) and (3.7), we get

$$
\Upsilon=\sum_{n=0}^{\infty} B_{n, \lambda, \lambda_{1}, \lambda_{2}, \lambda_{3}}^{[j]}(1-z: \omega, x, y) \frac{t^{n}}{n !} \sum_{n=0}^{\infty} S_{2, \lambda_{1}, \omega}(n, k) \frac{t^{n}}{n !}(z)_{k, \lambda}
$$




$$
=\sum_{n=0}^{\infty}\left(\sum_{u=0}^{n}\left(\begin{array}{l}
n \\
u
\end{array}\right) B_{u, \lambda, \lambda_{1}, \lambda_{2}, \lambda_{3}}^{[j]}(1-z: \omega, x, y) S_{2, \lambda_{1}, \omega}(n-u, k)(z)_{k, \lambda}\right) \frac{t^{n}}{n !} .
$$

Thus, we arrive at the following theorem.

Theorem 4.2. The following summation equality

$$
\sum_{u=0}^{n} \mathfrak{B}_{k, u}(z: \lambda) S_{2, \lambda_{1}, \lambda_{2}, \lambda_{3}}^{[j, w]}(n, u: x, y)=\sum_{u=0}^{n}\left(\begin{array}{l}
n \\
u
\end{array}\right) B_{u, \lambda, \lambda_{1}, \lambda_{2}, \lambda_{3}}^{[j]}(1-z: \omega, x, y) S_{2, \lambda_{1}, \omega}(n-u, k)(z)_{k, \lambda}
$$

is valid.

The classical Bernoulli $B_{n}(x)$, Euler $E_{n}(x)$ and Genocchi $G_{n}(x)$ polynomials and the degenerate Bernoulli $B_{n, \lambda}(x)$, Euler $E_{n, \lambda}(x)$ and Genocchi $G_{n, \lambda}(x)$ polynomials are given as follows $(c f .[2,8,10,13$, $14,16,17,28,29,33-36])$ :

$$
\begin{aligned}
& \sum_{n=0}^{\infty} B_{n}(x) \frac{t^{n}}{n !}=\frac{t}{e^{t}-1} \text { and } \sum_{n=0}^{\infty} B_{n, \lambda}(x) \frac{t^{n}}{n !}=\frac{t}{e_{\lambda}(t)-1} e_{\lambda}^{x}(t), \\
& \sum_{n=0}^{\infty} E_{n}(x) \frac{t^{n}}{n !}=\frac{2}{e^{t}+1} \text { and } \sum_{n=0}^{\infty} E_{n, \lambda}(x) \frac{t^{n}}{n !}=\frac{2}{e_{\lambda}(t)+1} e_{\lambda}^{x}(t), \\
& \sum_{n=0}^{\infty} G_{n}(x) \frac{t^{n}}{n !}=\frac{2 t}{e^{t}+1} \text { and } \sum_{n=0}^{\infty} G_{n, \lambda}(x) \frac{t^{n}}{n !}=\frac{2 t}{e_{\lambda}(t)+1} e_{\lambda}^{x}(t) .
\end{aligned}
$$

We here generalize the mentioned polynomials above via the generalized degenerate Gould-Hopper polynomials in (1.6).

Definition 4.3 ([11]). the following exponential generating functions are the definition of the generalized degenerate Gould-Hopper based degenerate Bernoulli $B_{n, \lambda_{1} ; \lambda_{2}, \lambda_{3}}^{[j]}(x, y)$, Euler $E_{n, \lambda_{1} ; \lambda_{2}, \lambda_{3}}^{[j]}(x, y)$ and Genocchi $G_{n, \lambda_{1} ; \lambda_{2}, \lambda_{3}}^{[j]}(x, y)$ polynomials:

$$
\begin{aligned}
& \sum_{n=0}^{\infty} B_{n, \lambda_{1} ; \lambda_{2}, \lambda_{3}}^{[j]}(x, y) \frac{t^{n}}{n !}=\frac{t}{e_{\lambda_{1}}(t)-1} e_{\lambda_{2}}^{x}(t) e_{\lambda_{3}}^{y}\left(t^{j}\right), \\
& \sum_{n=0}^{\infty} E_{n, \lambda_{1} ; \lambda_{2}, \lambda_{3}}^{[j]}(x, y) \frac{t^{n}}{n !}=\frac{2}{e_{\lambda_{1}}(t)+1} e_{\lambda_{2}}^{x}(t) e_{\lambda_{3}}^{y}\left(t^{j}\right), \\
& \sum_{n=0}^{\infty} G_{n, \lambda_{1} ; \lambda_{2}, \lambda_{3}}^{[j]}(x, y) \frac{t^{n}}{n !}=\frac{2 t}{e_{\lambda_{1}}(t)+1} e_{\lambda_{2}}^{x}(t) e_{\lambda_{3}}^{y}\left(t^{j}\right)
\end{aligned}
$$

for $\lambda_{1}, \lambda_{2}, \lambda_{3} \in \mathbb{R} \backslash\{0\}$.

When $x=y=0$, the polynomials in (4.6), (4.7), and (4.8) reduce to the corresponding degenerate numbers, namely $B_{n, \lambda_{1} ; \lambda_{2}, \lambda_{3}}^{[j]}(0,0):=B_{n, \lambda_{1}}, E_{n, \lambda_{1} ; \lambda_{2}, \lambda_{3}}^{[j]}(0,0):=E_{n, \lambda_{1}}$ and $G_{n, \lambda_{1} ; \lambda_{2}, \lambda_{3}}^{[j]}(0,0):=G_{n, \lambda_{1}}$, see $[8,29]$ and the references cited therein for further information. Several properties and relations of these polynomials have been proved by Duran and Acikgoz in [11].

Remark 4.4. When $y=0$ and $\lambda_{2}=\lambda_{1}$, the polynomials in (4.6), (4.7) and (4.8) reduce to the degenerate polynomials given in (4.3), (4.4), and (4.5).

We now perform to acquire several representations for $B_{n, \lambda, \lambda_{1}, \lambda_{2}, \lambda_{3}}^{[j]}(z: \omega, x, y)$ by means of the generalized degenerate Gould-Hopper based degenerate Bernoulli, Euler and Genocchi polynomials and fully degenerate Bell polynomials.

We here provide a relation involving the polynomials $B_{n, \lambda, \lambda_{1}, \lambda_{2}, \lambda_{3}}^{[j]}(z: \omega, x, y), B_{n, \lambda, \lambda_{1}}(x: \omega)$, and $B_{n, \lambda_{1} ; \lambda_{2}, \lambda_{3}}^{[j]}(x, y)$ as follows. 
Theorem 4.5. The following correlation

$$
B_{n, \lambda, \lambda_{1}, \lambda_{2}, \lambda_{3}}^{[j]}(z: \omega, x, y)=\sum_{u=0}^{n} \sum_{s=0}^{u}\left(\begin{array}{l}
n \\
u
\end{array}\right)\left(\begin{array}{l}
u \\
s
\end{array}\right) B_{u-s, \lambda, \lambda_{1}}(z: \omega) B_{s, \lambda_{1} ; \lambda_{2}, \lambda_{3}}^{[j]}(x, y) \frac{(1)_{n-u+1, \lambda_{1}}}{n-u+1}
$$

holds true.

Proof. By (3.7) and (4.6), we get

$$
\begin{aligned}
& \sum_{n=0}^{\infty} B_{n, \lambda, \lambda_{1}, \lambda_{2}, \lambda_{3}}^{[j]}(z: \omega, x, y) \frac{t^{n}}{n !} \\
& \quad=e_{\lambda}^{z}\left(e_{\lambda_{1}}^{\omega}(t)-1\right) e_{\lambda_{2}}^{x}(t) e_{\lambda_{3}}^{y}\left(t^{j}\right) \frac{t}{e_{\lambda_{1}}(t)-1} \frac{e_{\lambda_{1}}(t)-1}{t} \\
& \quad=\sum_{n=0}^{\infty} B_{n, \lambda, \lambda_{1}}(z: \omega) \frac{t^{n}}{n !} \sum_{n=0}^{\infty} B_{n, \lambda_{1} ; \lambda_{2}, \lambda_{3}}^{[j]}(x, y) \frac{t^{n}}{n !} \sum_{n=0}^{\infty}(1)_{n+1, \lambda_{1}} \frac{t^{n}}{(n+1) !} \\
& \quad=\sum_{n=0}^{\infty}\left(\sum_{u=0}^{n}\left(\begin{array}{l}
n \\
u
\end{array}\right) \sum_{s=0}^{u}\left(\begin{array}{l}
u \\
s
\end{array}\right) B_{u-s, \lambda, \lambda_{1}}(z: \omega) B_{s, \lambda_{1} ; \lambda_{2}, \lambda_{3}}^{[j]}(x, y) \frac{(1)_{n-u+1, \lambda_{1}}}{n-u+1}\right) \frac{t^{n}}{n !},
\end{aligned}
$$

which implies the desired result (4.9).

We give the following theorem.

Theorem 4.6. The following summation formula

$$
\begin{aligned}
B_{n, \lambda, \lambda_{1}, \lambda_{2}, \lambda_{3}}^{[j]}(z: \omega, x, y)= & \sum_{k=0}^{n} \sum_{m=0}^{k}\left(\begin{array}{l}
n \\
k
\end{array}\right)\left(\begin{array}{l}
k \\
m
\end{array}\right) \frac{(1)_{n-k, \lambda_{1}}}{2} B_{k-m, \lambda, \lambda_{1}}(z: \omega) E_{m, \lambda_{1} ; \lambda_{2}, \lambda_{3}}^{[j]}(x, y) \\
& +\frac{1}{2} \sum_{k=0}^{n}\left(\begin{array}{l}
n \\
k
\end{array}\right) B_{n-k, \lambda, \lambda_{1}}(z: \omega) E_{k, \lambda_{1} ; \lambda_{2}, \lambda_{3}}^{[j]}(x, y)
\end{aligned}
$$

is valid.

Proof. From (3.7) and (4.7), the aimed result (4.10) can be directly obtained by utilizing similar method used in the proof of Theorem 4.2. Thus, we omit the proof.

A correlation covering the generalized degenerate Gould-Hopper based degenerate Genocchi polynomials and the fully degenerate Bell polynomials is stated in the following theorem.

Theorem 4.7. The following relation

$$
\begin{aligned}
B_{n, \lambda, \lambda_{1}, \lambda_{2}, \lambda_{3}}^{[j]}(z: \omega, x, y)= & \frac{1}{n+1} \sum_{k=0}^{n+1} \sum_{m=0}^{k}\left(\begin{array}{c}
n+1 \\
k
\end{array}\right)\left(\begin{array}{c}
k \\
m
\end{array}\right) \frac{(1)_{n+1-k, \lambda_{1}}}{2} B_{k-m, \lambda, \lambda_{1}}(z: \omega) G_{m, \lambda_{1} ; \lambda_{2}, \lambda_{3}}^{[j]}(x, y) \\
& +\sum_{k=0}^{n+1}\left(\begin{array}{c}
n+1 \\
k
\end{array}\right) \frac{B_{n+1-k, \lambda, \lambda_{1}}(z: \omega) G_{k, \lambda_{1} ; \lambda_{2}, \lambda_{3}}^{[j]}(x, y)}{2(n+1)}
\end{aligned}
$$

holds true.

Proof. In view of (3.7) and (4.8), the proof can be directly attained by utilizing similar method used in the proof of Theorem 4.2. Thus, we omit the proof. 


\section{References}

[1] P. Appell, J. Kampé de Fériet, Fonctions Hypergéométriques et Hypersphériques: Polynômes d'Hermite, Gauthier-Villars, Paris, (1926). 1, 2, 7, 3, 3, 7

[2] S. Araci, W. A. Khan, M. Acikgoz, C. Özel, P. Kumam, A new generalization of Apostol type Hermite-Genocchi polynomials and its applications, SpringerPlus, 5 (2016), 17 pages. 1, 3, 3, 7, 4

[3] E. T. Bell, Exponential polynomials, Ann. of Math. (2), 35 (1934), 258-277. 1, 1, 1, 1, 3

[4] Y. Ben Cheikh, A. Zaghouani, Some discrete d-orthogonal polynomials sets, J. Comput. Appl. Math., 156 (2003), 253-263. 2, 7

[5] S. Bouroubi, M. Abbas, New identities for Bell's polynomials: New approaches, Rostock. Math. Kolloq., 61 (2006), 49-55. 1, 1

[6] F. E. Browder, W. V. Petryshyn, Construction of fixed points of nonlinear mappings in Hilbert spaces, J. Math. Anal. Appl., 20 (1967), 197-228.

[7] L. Carlitz, A degenerate Staudt-Clausen theorem, Arch. Math. (Basel), 7 (1956), 28-33. 1, 1, 2, 7

[8] L. Carlitz, Degenerate Stirling, Bernoulli and Eulerian numbers, Utilitas Math., 15 (1979), 51-88. 4, 4

[9] L. Carlitz, Some remarks on the Bell numbers, Fibonacci Quart., 18 (1980), 66-73.

[10] G. Dattoli, S. Lorenzutta, C. Cesarano, Finite sums and generalized forms of Bernoulli polynomials, Rend. Mat. Appl. (7), 19 (1999), 385-391. 1, 1, 1.1, 1, 2, 7, 4

[11] U. Duran, M. Acikgoz, Generalized Gould-Hopper based fully degenerate central Bell polynomials, Turkish J. Anal. Number Theory, 7 (2019), 124-134. 1, 1, 1, 1.1, 1, 3, 6, 4.3, 4

[12] U. Duran, M. Acikgoz, On some degenerate probability distributions related to the degenerate Stirling numbers of the second kind, (under review). 1, 2.5, 6, 3, 3

[13] U. Duran, M. Acikgoz, S. Araci, Hermite based poly-Bernoulli polynomials with a q-parameter, Adv. Stud. Contemp. Math., 28 (2018), 285-296. 1, 4

[14] U. Duran, P. N. Sadjang, On Gould-Hopper-based fully degenerate poly-Bernoulli polynomials with a q-parameter, Mathematics, 7 (2019), 14 pages. 1, 1, 1, 1, 1.1, 1, 3, 3, 7, 4

[15] F. T. Howard, Bell polynomials and degenerate Stirling numbers, Rend. Sem. Mat. Univ. Padova, 61 (1979), $203-219$. 3,7

[16] F. T. Howard, Explicit formulas for degenerate Bernoulli numbers, Discrete Math., 162 (1996), 175-185. 1, 1, 1, 1, 3, 3, 4

[17] W. A. Khan, A note on degenerate Hermite poly-Bernoulli numbers and polynomials, J. Class. Anal., 8 (2016), 65-76. 1, $1,1,1,2,7,4$

[18] W. A. Khan, K. S. Nisar, S. Araci, M. Acikgoz, Fully degenerate Hermite poly Bernoulli numbers and polynomials, Adv. Appl. Math. Sci., 17 (2018), 461-478.

[19] T. Kim, A note on degenerate Stirling polynomials of the second kind, Proc. Jangjeon Math. Soc., 20 (2017), 319-331. 2, $6,3,3$

[20] D. S. Kim, T. Kim, Some identities of Bell polynomials, Sci. China Math., 58 (2015), 2095-2104. 1, 1, 1, 1, 2, 7, 3, 7

[21] T. Kim, D. S. Kim, On degenerate Bell numbers and polynomials, Rev. R. Acad. Cienc. Exactas Fís. Nat. Ser. A Mat. RACSAM, 111 (2017), 435-446. 2, 3, 4, 6

[22] T. Kim, D. S. Kim, Degenerate Bernstein polynomials, Rev. R. Acad. Cienc. Exactas Fís. Nat. Ser. A Mat. RACSAM, 113 (2019), 2913-2920. 2, 7, 3, 3, 4

[23] T. Kim, D. S. Kim, D. V. Dolgy, On partially degenerate Bell numbers and polynomials, Proc. Jangjeon Math. Soc., 20 (2017), 337-345. 1, 1, 1, 1, 3, 3, 6

[24] T. Kim, D. S. Kim, G.-W. Jang, Extended Stirling polynomials of the second kind and extended Bell polynomials, Proc. Jangjeon Math. Soc., 20 (2017), 365-376. 6

[25] T. Kim, D. S. Kim, L.-C. Jang, H.-I. Kwon, Extended degenerate stirling numbers of the second kind and extended degenerate Bell polynomials, Util. Math., 106 (2018), 11-21. 1, 1, 1, 1, 2, 2, 5, 7, 3, 3, 6

[26] T. Kim, D. S. Kim, H. Y. Kim, J. Kwon, Degenerate Stirling polynomials of the second kind and some applications, Symmetry, 11 (2019), 11 pages. 3, 3, 7

[27] T. Kim, Y. Yao, D. S. Kim, G.-W. Jang, Degenerate x-Stirling numbers and x-Bell polynomials, Russ. J. Math. Phys., 25 (2018), 44-58. 1, 1, 1, 1, 2, 2, 5, 6, 7, 3, 3, 6

[28] B. Kurt, Y. Simsek, On the Hermite based Genocchi polynomials, Adv. Stud. Contemp. Math. (Kyungshang), 23 (2013), 13-17. 1, 1, 4

[29] D. Lim, Some identities of degenerate Genocchi polynomials, Bull. Korean Math. Soc., 53 (2016), 569-579. 2, 7, 4, 4

[30] M. Mihoubi, Bell polynomials and binomial type sequences, Discret Math., 308 (2008), 2450-2459.

[31] S. P. Njionou, Addition theorems for the $\Delta_{\omega}$-Appell polynomials and the associated classes of polynomial expansions, (under review). 1, 1, 1.1, 3, 3

[32] B. O'Neill, Semi-Riemannian geomerty with applications to relativity, Academic Press, London, (1983). 3, 3, 7

[33] M. A. Özarslan, Hermite-based unified Apostol-Bernoulli, Euler and Genocchi polynomials, Adv. Difference Equ., 2013 (2013), 13 pages. 1, 1, 1, 2, 7, 4

[34] M. A. Pathan, A new class of generalized Hermite-Bernoulli polynomials, Georgian Math. J., 19 (2012), 559-573. 1

[35] E. D. Rainville, Special functions, Macmillan Co., New York, (1960). 
[36] H. M. Srivastava, J. S. Choi, Zeta and q-Zeta functions and associated series and integrals, Elsevier, Amsterdam, (2012). 4

[37] D. V. Widder, The Heat Equation, Academic Press, New York-London, (1975). 1

[38] Y. H. Yao, Y. J. Cho, Y.-C. Liou, R. P. Agarwal, Constructed nets with perturbations for equilibrium and fixed point problems, J. Inequal. Appl., 2014 (2014), 14 pages. 2, 7 